\title{
Application value of MRI diffuse weighted imaging combined with PET/CT in the diagnosis of stomach cancer at different stages
}

\author{
YANBIN SUI ${ }^{1 *}$, ZHENXING ZOU $^{1 *},{\text { FANGFANG } \mathrm{LI}^{2} \text { and CUIJUAN HAO }}^{1}$ \\ Departments of ${ }^{1}$ Medical Image and ${ }^{2}$ Blood Purification, The Affiliated Yantai Yuhuangding Hospital of \\ Qingdao University, Yantai, Shandong 264000, P.R. China
}

Received August 23, 2018; Accepted April 17, 2019

DOI: 10.3892/ol.2019.10286

\begin{abstract}
Value of MRI diffusion-weighted imaging (MRI DWI) combined with PET/CT in the diagnosis and staging of stomach cancer (SC) was investigated. A retrospective analysis was performed on 160 patients with SC diagnosed by pathological biopsy in The Affiliated Yantai Yuhuangding Hospital of Qingdao University from March 2015 to April 2018. The values of MRI DWI, PET/CT and combined diagnosis in the diagnosis and staging of SC were compared according to the criteria of diagnosis of postoperative pathological or clinical comprehensive evaluation. The sensitivity, specificity and diagnostic coincidence rate of MRI DWI in the diagnosis of SC at stage I-II were 61.05, 64.62 and $62.50 \%$, respectively, which were significantly lower than those of PET/CT $(\mathrm{P}<0.05)$. Sensitivity, specificity and diagnostic coincidence rate of MRI DWI in the diagnosis of $\mathrm{SC}$ at stage III-IV were lower than those of PET/CT $(\mathrm{P}<0.05)$. Sensitivity and diagnostic coincidence rate of MRI DWI combined with PET/CT in the diagnosis of SC at stage I-II were significantly higher than those of MRI DWI or PET/CT alone $(\mathrm{P}<0.05)$. Specificity and diagnostic coincidence rate of MRI DWI combined with PET/CT in the diagnosis of SC at stage III-IV were significantly higher than those of MRI DWI or PET/CT alone $(\mathrm{P}<0.05)$. PET/CT is superior to MRI DWI in SC staging, whereas the diagnostic efficiency of combined scan is much higher than that of PET/CT or MRI DWI alone.
\end{abstract}

Correspondence to: Dr Cuijuan Hao, Department of Medical Image, The Affiliated Yantai Yuhuangding Hospital of Qingdao University, 20 Yuhuangding East Road, Yantai, Shandong 264000, P.R. China

E-mail: ey36ga@163.com

Dr Fangfang Li, Department of Blood Purification, The Affiliated Yantai Yuhuangding Hospital of Qingdao University, 20 Yuhuangding East Road, Yantai, Shandong 264000, P.R. China

E-mail: hfw9ae@163.com

*Contributed equally

Key words: MRI diffusion-weighted imaging, PET/CT, stomach cancer, diagnosis, staging
In order to obtain more accurate preoperative staging and to avoid diagnostic exploratory laparotomy, the combination of MRI DWI and PET/CT techniques should be used in the comprehensive analysis of the disease to improve the accuracy of clinical diagnosis.

\section{Introduction}

Stomach cancer (SC), a malignant gastric cancer, originate from the most superficial mucosal epithelial cells of the gastric wall (1). According to the report released by the World Health Organization (WHO), the annual incidence of SC in the world is $14.23 / 100,000$, and more than one million new SC are diagnosed every year in the world. The incidence rate of SC increases significantly with the increase of age, and the peak age range of the disease is 49-80 years, showing a younger trend. Purpose of SC staging is to evaluate the onset of the disease, to facilitate clinicians to summarize and communicate therapeutic effects, to conduct collaborative research on SC, and to develop treatment regimens (2-4). Although pathological diagnosis is the golden standard of clinical staging of SC, some patients can not accept it psychologically and physiologically (5). Due to the development and innovation of medical diagnostic method, the imaging techniques used in clinical diagnosis are constantly upgraded, and the diagnostic coincidence rate is more and more close to pathological diagnosis. At present, M.R.I. diffuse weighted imaging (MRI DWI) and positron emission tomography/computed tomography (PET/CT) are new imaging techniques commonly used in SC clinical staging $(6,7)$.

MRI is an advanced imaging device that has no radiation effects on the human, and can perform local and systemic scans. MRI DWI is a new MR imaging technique fused DWI on the basis of MRI (8). PET/CT, a scanner combined by positron emission tomography and X-ray computed tomography, merges the two imaging techniques perfectly to gain complementary advantages. PET images provide molecular information such as function and metabolism, and CT provides detailed anatomical and pathological information. Pathophysiological and morphological changes of the disease can be reflected by the fusion of these two techniques (9-11). As an advanced examination method at present, the clinical value of PET/CT in the differential diagnosis of tumors, especially SC, cannot be ignored. In addition, it is also non-invasive (12). In this study, 
the application value of MRI DWI combined with PET/CT in the diagnosis of SC in different stages was evaluated.

\section{Patients and methods}

Inclusion and data collection. One hundred and sixty patients with SC diagnosed by pathological biopsy in The Affiliated Yantai Yuhuangding Hospital of Qingdao University (Yantai, China) from March 2015 to April 2018 were analyzed retrospectively. One hundred and two males and 58 females were included in the study, with an age range of 25-80 years, and a mean age of $50.46 \pm 29.54$ years (Table I).

Inclusion criteria: i) Only SC patients admitted to The Affiliated Yantai Yuhuangding Hospital of Qingdao University, and tissue samples examined as SC by combined examination of general surgery and pathology were included; and ii) patients who had not received radiotherapy, chemotherapy or other treatment. Exclusion criteria: i) Pregnant women and patients with allergic reactions to contrast agents, claustrophobia and other contraindications.

The study was approved by the Ethics Committee of The Affiliated Yantai Yuhuangding Hospital of Qingdao University. Patients who participated in this research had complete clinical data. Signed informed consents were obtained from the patients or the guardians.

Main reagents and instruments. Siemens Verio 3.0T superconducting magnetic resonance instrument was purchased from Siemens AG (Munich, Germany). The bolus injection contrast agent gadopentetate dimeglumine (Gd-DTPA) was purchased from Accdon Company (Waltham, MA, USA). PET/CT imaging agents: ${ }^{18} \mathrm{~F}$-deoxyglucose $\left({ }^{18} \mathrm{FDG}\right)$ was purchased from Accdon Company. PET/CT scanner was purchased from Royal Philips Electronics co., Ltd. (Amsterdam, The Netherlands). A 64-slice spiral CT was purchased from Siemens AG.

MRI-DWI examination methods (13). i) The subjects did not eat dinner the day before the examination, and the next day, $250 \mathrm{~g}$ of saline was injected from the anus of the subjects before 9 a.m.

ii) Eight channel Torso phased-array body coil was placed in the lower abdomen, and a pad was fixed between the coil and the lower abdomen. The center of the coil was located at $5 \mathrm{~cm}$ above the pubic symphysis. Imaging sequence: T1WI-weighted images of SE sagittal position and T2WI-weighted images of CSE sagittal position were generated first; TR: $250-4,000 \mathrm{msec} /$ 2,000-3,000 msec; Slice thickness: 3-5 mm, interval: 0.2-0.3 mm, TE: 10-20/100-120 msec; cross-sectional T1WI and T2WI weighted images were generated with the same imaging parameters as above. Then an enhanced examination was performed and $0.2 \mathrm{ml} / \mathrm{kg}$ Gd-DTPA was injected via anterior cubital vein at a rate of $2.5 \mathrm{ml} / \mathrm{sec}$. After injection, the conduit was cleaned with $30 \mathrm{ml}$ of saline.

iii) The axial and sagittal images of the lesion were generated by the thin-section images of gradient echo 3D T1-weigted imaging. TE: $7 \mathrm{msec}$; TR: $15 \mathrm{msec}$, slice thickness: $2 \mathrm{~mm}$. Diffuse weighted sequence: Axial TR: 4,000 msec; Matrix: 320x224; TE: 62.3-75.6 msec; slice thickness: $6 \mathrm{~mm}$; FOV: $38 \mathrm{~cm}$ x $22.8 \mathrm{~cm}$; slice gap: $2 \mathrm{~mm}$; NEX: 6; B value: $1,200 \mathrm{sec} / \mathrm{mm}^{2}$.
Table I. General clinical data of 160 patients with SC.

\begin{tabular}{|c|c|}
\hline Factors & $\mathrm{n}(\%)$ \\
\hline \multicolumn{2}{|l|}{ Sex } \\
\hline Male & $102(63.75)$ \\
\hline Female & $58(36.25)$ \\
\hline \multicolumn{2}{|l|}{ Age (years) } \\
\hline$\leq 50$ & $65(40.63)$ \\
\hline$>50$ & $95(59.37)$ \\
\hline \multicolumn{2}{|l|}{ Smoking } \\
\hline Yes & $110(68.75)$ \\
\hline No & $50(31.25)$ \\
\hline \multicolumn{2}{|l|}{ SC histological classification } \\
\hline Adenocarcinoma & $98(61.25)$ \\
\hline Adenosquamous carcinoma & $13(8.13)$ \\
\hline Squamous cell carcinoma & $34(21.25)$ \\
\hline Carcinoid & $15(9.37)$ \\
\hline \multicolumn{2}{|l|}{ SC clinical staging } \\
\hline Stage I & $60(37.50)$ \\
\hline Stage II & $35(21.88)$ \\
\hline Stage III & $40(25.00)$ \\
\hline Stage IV & $25(15.62)$ \\
\hline \multicolumn{2}{|l|}{ Cell differentiation degree } \\
\hline Well-differentiated & $75(46.88)$ \\
\hline Moderately differentiated & $40(25.00)$ \\
\hline Poorly differentiated & $45(28.12)$ \\
\hline \multicolumn{2}{|l|}{ Lymphatic metastasis } \\
\hline Yes & $55(34.37)$ \\
\hline No & $105(65.63)$ \\
\hline
\end{tabular}

Method of PET/CT examination. i) Establishment of the weight of the patient (the injection measurement of image agent should be controlled according to patient's weight).

ii) Detection of blood glucose in SC patients: Patients with $\mathrm{SC}$ should fast for at least $6 \mathrm{~h}$ before examination. After $6 \mathrm{~h}$, the venous blood glucose concentration of SC patients was measured to ensure that the blood glucose concentration was $<7.8 \mathrm{mmol} / \mathrm{l}$. Too high or too low blood glucose concentration should be handled in time.

iii) Injection of PET/CT imaging agent: ${ }^{18} \mathrm{~F}-\mathrm{FDG}$ imaging agent was injected into patient's elbow vein when patient's blood glucose concentration was within the normal range. The radiochemical purity should be $>95 \%$.

iv) Performing PET/CT examination: Patients needed to empty their urine first and then drink $600 \mathrm{ml}$ purified water before PET/CT examination. CT transmission scanning was performed on the lesions of SC patients first, and the PET was used to scan the largest range of SV lesions, then the decay data of CT was corrected. The fusion images of CT, PET and $\mathrm{PET} / \mathrm{CT}$ in all directions were then formed.

Criteria of judgement. i) Image analysis of MRI DWI in SC (Table II); and ii) Image analysis of PET/CT in SC staging (Table III). 
Table II. Image features of MRI DWI in different stages of SC.

\begin{tabular}{ll}
\hline Stages & \multicolumn{1}{c}{ Image features } \\
\hline I & Lesion is located in mucosa and submucosa \\
II & Tumor reaches muscular layer \\
III & The tumor penetrates the muscular layer to \\
& the peripheral fat \\
IV & Other organs are invaded by tumor cells
\end{tabular}

Table III. Image features of PET/CT in different stages of SC.

\begin{tabular}{ll}
\hline Stages & \multicolumn{1}{c}{ Image features } \\
\hline I & $\begin{array}{l}\text { Invading of mucoderm or muscularis mucosae, } \\
\text { submucosa } \\
\text { Invading of muscularis propria }\end{array}$ \\
II & $\begin{array}{l}\text { Penetrating subserous connective tissue } \\
\text { III }\end{array}$ \\
IV & $\begin{array}{l}\text { anfiltrating the serous membrane or invading } \\
\text { adjacent organs }\end{array}$ \\
\hline
\end{tabular}

Statistical analysis. SPSS 17.0 (SPSS, Inc., Chicago, IL, USA) software system was used for statistical analysis. The enumeration data were presented as [n (\%)]. $\chi^{2}$ test was used for the univariate analysis of diagnostic accuracy of SC at different stages. $\mathrm{P}<0.05$ was considered to indicate a statistically significant difference.

\section{Results}

Diagnostic efficacy of MRI DWI and PET/CT in SC at different stages. i) Diagnostic efficacy of MRI DWI and $\mathrm{PET} / \mathrm{CT}$ in SC at stage I-II. The sensitivity, specificity and diagnostic accordance rate of MRI DWI in the diagnosis of SC at stage I-II were $61.05,64.62$ and $62.50 \%$, respectively. The sensitivity, specificity and diagnostic accordance rate of PET/CT in the diagnosis of SC at stage I-II were 85.26, 81.54 and $83.75 \%$, respectively. Comparing the data in the two groups, it was showed that the sensitivity, specificity and diagnostic coincidence rate of MRI DWI in the diagnosis of SC at stage I-II were significantly lower than those of PET/CT, and the difference was statistically significant $(\mathrm{P}<0.05)$ (Tables IV-VI).

ii) Diagnostic efficacy of MRI DWI and PET/CT in SC at stage III-IV. The sensitivity, specificity and diagnostic accordance rate of MRI DWI in the diagnosis of SC at stage III-IV were $80.00,71.58$ and $75.00 \%$, respectively. The sensitivity, specificity and diagnostic accordance rate of PET/CT in the diagnosis of SC at stage III-IV were 81.54, 85.26 and $83.75 \%$, respectively. Comparing the data in the two groups showed that the sensitivity, specificity and diagnostic coincidence rate of MRI DWI in the diagnosis of SC at stage III-IV were significantly lower than those of PET/CT. The difference of specificity was statistically significant $(\mathrm{P}<0.05)$, and there was no significant difference in sensitivity and diagnostic coincidence rate between the two groups ( $\mathrm{P}>0.05)$ (Tables VII-IX).
Table IV. Diagnostic efficacy of MRI DWI in SC at I-II stage.

\begin{tabular}{lccc}
\hline & \multicolumn{2}{c}{ Results of pathological diagnosis } & \\
\cline { 2 - 3 } Groups & I-II stage & Non-I-II stage & Total \\
\hline $\begin{array}{l}\text { MRI DWI diagnosis } \\
\text { of I-II stage }\end{array}$ & 58 & 23 & 81 \\
$\begin{array}{l}\text { MRI DWI diagnosis } \\
\text { of non-I-II stage }\end{array}$ & 37 & 42 & 79 \\
\begin{tabular}{l} 
Total \\
\hline
\end{tabular} & 95 & 65 & 160 \\
\hline
\end{tabular}

Table V. Diagnostic efficacy of PET/CT in SC at I-II stage.

\begin{tabular}{lccc}
\hline & \multicolumn{2}{c}{ Results of pathological diagnosis } & \\
\cline { 2 - 3 } Groups & I-II stage & Non-I-II stage & Total \\
\hline $\begin{array}{l}\text { PET/CT diagnosis } \\
\text { of I-II stage }\end{array}$ & 81 & 12 & 93 \\
$\begin{array}{l}\text { PET/CT diagnosis } \\
\text { of non-I-II stage }\end{array}$ & 14 & 53 & 67 \\
\begin{tabular}{l} 
Total \\
\hline
\end{tabular} & 95 & 65 & 160 \\
\hline
\end{tabular}

Comparison of diagnostic efficacy between MRI DWI combined with PET/CT and MRI DWI or PET/CT alone at different SC stages. The sensitivity, specificity and diagnostic coincidence rate of MRI DWI combined with PET/CT in the diagnosis of SC at stage I-II were $91.58,80.00$ and $86.88 \%$, respectively. The sensitivity and diagnostic coincidence rate of MRI DWI combined with PET/CT in the diagnosis of SC at stage I-II were significantly higher than those of MRI DWI or PET/CT alone, and the differences were statistically significant $(\mathrm{P}<0.05)$. While the sensitivity, specificity and diagnostic coincidence rate of MRI DWI combined with PET/CT in the diagnosis of SC at stage III-IV were $80.00,91.58$ and $86.88 \%$, respectively. The sensitivity and diagnostic coincidence rate of MRI DWI combined with PET/CT in the diagnosis of SC at stage III-IV were significantly higher than those of MRI DWI or $\mathrm{PET} / \mathrm{CT}$ alone, and the differences were statistically significant $(\mathrm{P}<0.05)$ (Tables X-XII).

\section{Discussion}

Targeted therapy is very important to SC, the key of which is the early detection and accurate staging of SC (14). With the continuous progress and innovation of medical science and technology, the imaging equipment and technology of medical imaging are also making continuous progress. Both MRI DWI and PET/CT are new medical imaging techniques based on traditional MRI, DWI, CT and PET, and have been widely used in early diagnosis, clinical staging and monitoring of curative effect. There is little difference in the economic burden to patients between MRI DWI and PET/CT. Different medical imaging techniques have different imaging principles, and each imaging technique has its own clinical application scope and unique performance. But up to now, there is no definitional judgment that one image 
Table VI. Comparison of the diagnostic efficacy of MRI DWI and PET/CT in SC at I-II stage.

\begin{tabular}{lllrr}
\hline Factors & \multicolumn{1}{c}{ MRI DWI } & \multicolumn{1}{c}{ PET/CT } & \multicolumn{1}{c}{$\chi^{2}$} & P-value \\
\hline Sensitivity & $61.05 \%(58 / 95)$ & $85.26 \%(81 / 95)$ & 14.180 & $<0.001$ \\
Specificity & $64.62 \%(42 / 65)$ & $81.54 \%(53 / 65)$ & 4.731 & 0.030 \\
Diagnostic accordance rate & $62.50 \%(100 / 160)$ & $83.75 \%(134 / 160)$ & 18.380 & $<0.001$ \\
\hline
\end{tabular}

Table VII. Diagnostic efficacy of MRI DWI in SC at III-IV stage.

\begin{tabular}{lccr}
\hline & \multicolumn{2}{c}{ Results of pathological diagnosis } & \\
\cline { 2 - 3 } Groups & III-IV stage & Non-III-IV stage & Total \\
\hline MRI DWI diagnosis of III-IV stage & 52 & 27 & 79 \\
MRI DWI diagnosis of non-III-IV stage & 13 & 68 & 81 \\
Total & 65 & 95 & 160 \\
\hline
\end{tabular}

Table VIII. Diagnostic efficacy of PET/CT in SC at III-IV stage.

\begin{tabular}{lccr}
\hline & \multicolumn{2}{c}{ Results of pathological diagnosis } & \\
\cline { 2 - 4 } Groups & III-IV stage & Non-III-IV stage & Total \\
\hline PET/CT diagnosis of III-IV stage & 53 & 14 & 67 \\
PET/CT diagnosis of non-III-IV stage & 12 & 81 & 93 \\
Total & 65 & 95 & 160 \\
\hline
\end{tabular}

technique can completely replace another $(15,16)$. MRI DWI is an imaging technique developed on the basis of MRI (13), and $\mathrm{PET} / \mathrm{CT}$ is the most advanced and the best molecular imaging technique for early diagnosis, clinical staging and monitoring of curative effect of tumors and it has been widely used in clinical practice (17). However, there are still some differences in the indications, advantages and disadvantages of these two medical imaging techniques in tumor examination (18). This study explored the value of MRI DWI combined with PET/CT in the diagnosis of SC at different stages.

The diagnosticefficacy of MRIDWI and PET/CT in different SC stages was compared in this study. The results showed that the sensitivity, specificity and diagnostic coincidence rate of MRI DWI in the diagnosis of SC at stage I-II were 61.05, 64.62 and $62.50 \%$, respectively, which were significantly lower than those of PET/CT, and the difference between the two groups was statistically significant $(\mathrm{P}<0.05)$. The sensitivity, specificity and diagnostic coincidence rate of MRI DWI in the diagnosis of SC at stage III-IV were lower than those of PET/CT, and the difference of specificity between the two groups was statistically significant $(\mathrm{P}<0.05)$. The differences of sensitivity and diagnostic coincidence rate were not statistically significant $(\mathrm{P}>0.05)$. Therefore, it is concluded PET/CT is more effective than MRI DWI in the diagnosis of SC clinical staging, and is more suitable for the clinical diagnosis of SC at stage I-IV. There are few reports of MRI DWI and PET/CT in different stages of SC, but from the point of view of Stecco et al (19), the diagnostic efficacy of PET/CT in SC is better than that of MRI DWI, and it is more suitable for the clinical diagnosis of $\mathrm{SC}$ at stage I-II. By comparing the clinical application value of MRI DWI and PET/CT in tumors, Stecco et al found that the clinical diagnostic value of MRI DWI in diagnosis of brain tumors was higher than that in thoracic and abdominal tumors (19). A comparison of the diagnostic efficacy between MRI DWI combined with PET/CT and MRI DWI or PET/CT alone in different SC stages was made in this study. The results showed that the sensitivity and diagnostic coincidence rate of MRI DWI combined with PET/CT in the diagnosis of SC at stage I-II were significantly higher than those of MRI DWI or $\mathrm{PET} / \mathrm{CT}$ alone, and the difference was statistically significant $(\mathrm{P}<0.05)$. While the specificity and diagnostic coincidence rate of MRI DWI combined with PET/CT in the diagnosis of SC at stage III-IV were significantly higher than those of MRI DWI or PET/CT alone, and the difference was statistically significant $(\mathrm{P}<0.05)$. Although there is no study on the same experimental design as in this investigation, a large number of studies on MRI DW, PET/CT and their combination are similar to our research results, which corroborate the research viewpoint of this report (19-21).

In this study, because of the small number of subjects there may be contingency in the experimental results.

In conclusion, the diagnostic efficacy of PET/CT at stage I-II was higher than that of MRI DWI. When the two techniques were combined, the diagnostic sensitivity, specificity 
Table IX. Comparison of the diagnostic efficacy of MRI DWI and PET/CT in SC at III-IV stage.

\begin{tabular}{|c|c|c|c|c|}
\hline Factors & MRI DWI & $\mathrm{PET} / \mathrm{CT}$ & $\chi^{2}$ & P-value \\
\hline Sensitivity & $80.00 \%(52 / 65)$ & $81.54 \%(53 / 65)$ & 0.050 & 0.824 \\
\hline Specificity & $71.58 \%(68 / 95)$ & $85.26 \%(81 / 95)$ & 5.256 & 0.022 \\
\hline Diagnostic accordance rate & $75.00 \%(120 / 160)$ & $83.75 \%(134 / 160)$ & 3.741 & 0.053 \\
\hline
\end{tabular}

Table X. Diagnostic efficacy of MRI DWI combined with PET/CT in SC at I-II stage.

\begin{tabular}{lrrr}
\hline & \multicolumn{2}{c}{ Results of pathological diagnosis } & Non-I-II stage \\
\cline { 2 - 4 } Groups & I-II stage & 13 & Total \\
\hline Combined diagnosis of I-II stage & 87 & 52 & 100 \\
Combined diagnosis of non-I-II stage & 8 & 65 & 160 \\
Total & 95 & 50 \\
\hline
\end{tabular}

Table XI. Diagnostic efficacy of MRI DWI combined with PET/CT in SC at III-IV stage.

\begin{tabular}{lcrr}
\hline & \multicolumn{2}{c}{ Results of pathological diagnosis } \\
\cline { 2 - 4 } Groups & \multicolumn{1}{c}{ III-IV stage } & Non-III-IV stage & Total \\
\hline Combined diagnosis of III-IV stage & 52 & 8 & 100 \\
Combined diagnosis of non-III-IV stage & 13 & 87 & 95 \\
Total & 65 & 160 \\
\hline
\end{tabular}

Table XII. Comparison of diagnostic efficacy between combined diagnosis and MRI DWI or PET/CT alone.

\begin{tabular}{|c|c|c|c|c|}
\hline Factors & Combined & MRI DWI & $\mathrm{PET} / \mathrm{CT}$ & P-value \\
\hline \multicolumn{5}{|l|}{ Stage I-II } \\
\hline Sensitivity & $91.58 \%(87 / 95)$ & $61.05 \%(58 / 95)$ & $85.26 \%(81 / 95)$ & $<0.001$ \\
\hline Specificity & $80.00 \%(52 / 65)$ & $64.62 \%(42 / 65)$ & $81.54 \%(53 / 65)$ & 0.047 \\
\hline Diagnostic accordance rate & $86.88 \%(139 / 160)$ & $62.50 \%(100 / 160)$ & $83.75 \%(134 / 160)$ & $<0.001$ \\
\hline \multicolumn{5}{|l|}{ Stage III-IV } \\
\hline Sensitivity & $80.00 \%(52 / 65)$ & $80.00 \%(52 / 65)$ & $81.54 \%(53 / 65)$ & 0.968 \\
\hline Specificity & $91.58 \%(87 / 95)$ & $71.58 \%(68 / 95)$ & $85.26 \%(81 / 95)$ & $<0.001$ \\
\hline Diagnostic accordance rate & $86.88 \%(139 / 160)$ & $75.00 \%(120 / 160)$ & $83.75 \%(134 / 160)$ & 0.017 \\
\hline
\end{tabular}

and coincidence rate for different stages of SC were greatly improved. Therefore, it is believed that the MRI DWI combined with PET/CT is of great significance to the future development of medical imaging techniques.

\section{Acknowledgements}

Not applicable.

\section{Funding}

No funding was received.

\section{Availability of data and materials}

The datasets used and/or analyzed during the current study are available from the corresponding author on reasonable request.

\section{Authors' contributions}

YS and ZZ wrote the manuscript and were responsible for the MRI-DWI examination and analysis. FL and $\mathrm{CH}$ recorded and analyzed the PCT/CT results. YS and $\mathrm{CH}$ assisted with the statistical analysis. All authors read and approved the final manuscript. 


\section{Ethics approval and consent to participate}

The study was approved by the Ethics Committee of The Affiliated Yantai Yuhuangding Hospital of Qingdao University (Yantai, China). Patients who participated in this research had complete clinical data. Signed informed consents were obtained from the patients or the guardians.

\section{Patient consent for publication}

Not applicable.

\section{Competing interests}

The authors declare that they have no competing interests.

\section{References}

1. Balakrishnan M, George R, Sharma A and Graham DY: Changing trends in stomach cancer throughout the world. Curr Gastroenterol Rep 19: 36, 2017.

2. Kodera Y: The current state of stomach cancer surgery in the world. Jpn J Clin Oncol 46: 1062-1071, 2016.

3. Yako-Suketomo $\mathrm{H}$ and Katanoda K: Comparison of time trends in stomach cancer mortality (1990-2006) in the world, from the WHO mortality database. Jpn J Clin Oncol 39: 622-623, 2009.

4. Yamamoto S: Stomach cancer incidence in the world. Jpn J Clin Oncol 31: 471, 2001.

5. Lu J, Huang CM, Zheng CH, Li P, Xie JW, Wang JB and Lin JX: Analysis on the clinical and pathological features and prognosis of familial gastric cancer in South China population: A single-center study of 724 patients. J Oncol 2012: 641218, 2012.

6. Kudou M, Kosuga T, Kubota T, Okamoto K, Komatsu S, Shoda K, Konishi H, Shiozaki A, Fujiwara H, Arita T, et al: Value of preoperative PET-CT in the prediction of pathological stage of gastric cancer. Ann Surg Oncol 25: 1633-1639, 2018.

7. Joo I, Lee JM, Kim JH, Shin CI, Han JK and Choi BI: Prospective comparison of 3T MRI with diffusion-weighted imaging and MDCT for the preoperative TNM staging of gastric cancer. J Magn Reson Imaging 41: 814-821, 2015.

8. Low RN and Gurney J: Diffusion-weighted MRI (DWI) in the oncology patient: Value of breathhold DWI compared to unenhanced and gadolinium-enhanced MRI. J Magn Reson Imaging 25: 848-858, 2007.

9. Hansen AE, McEvoy F, Engelholm SA, Law I and Kristensen AT: FDG PET/CT imaging in canine cancer patients. Vet Radiol Ultrasound 52: 201-206, 2011.

10. Cohade C, Osman M, Pannu HK and Wahl RL: Uptake in supraclavicular area fat ('USA-Fat'): Description on ${ }^{18} \mathrm{~F}-\mathrm{FDG}$ PET/CT. J Nucl Med 44: 170-176, 2003.
11. Freitag MT, Radtke JP, Hadaschik BA, Kopp-Schneider A, Eder M, Kopka K, Haberkorn U, Roethke M, Schlemmer HP and Afshar-Oromieh A: Comparison of hybrid (68)Ga-PSMA PET/MRI and (68)Ga-PSMA PET/CT in the evaluation of lymph node and bone metastases of prostate cancer. Eur J Nucl Med Mol Imaging 43: 70-83, 2016.

12. Shin S, Pak K, Kim SJ, Kim H and Kim SJ: Pulmonary tumor embolism derived from stomach cancer observation with serial ${ }^{18}$ F-FDG PET/CT. Clin Nucl Med 40: 270-272, 2015.

13. Han K, Croke J, Foltz W, Metser U, Xie J, Shek T, Driscoll B, Ménard C, Vines D, Coolens C, et al: A prospective study of DWI, DCE-MRI and FDG PET imaging for target delineation in brachytherapy for cervical cancer. Radiother Oncol 120: 519-525, 2016.

14. Wang TT, Qian XP and Liu BR: Survivin: Potential role in diagnosis, prognosis and targeted therapy of gastric cancer. World J Gastroenterol 13: 2784-2790, 2007.

15. Jambor I, Kuisma A, Ramadan S, Huovinen R, Sandell M, Kajander S, Kemppainen J, Kauppila E, Auren J, Merisaari H, et al: Prospective evaluation of planar bone scintigraphy, SPECT, SPECT/CT, ${ }^{18} \mathrm{~F}-\mathrm{NaF}$ PET/CT and whole body $1.5 \mathrm{~T}$ MRI, including DWI, for the detection of bone metastases in high risk breast and prostate cancer patients: SKELETA clinical trial. Acta Oncol 55: 59-67, 2016

16. Ehman EC, Johnson GB, Villanueva-Meyer JE, Cha S, Leynes AP, Larson PEZ and Hope TA: PET/MRI: Where might it replace PET/CT? J Magn Reson Imaging 46: 1247-1262, 2017.

17. Mhlanga JC, Chirindel A, Lodge MA, Wahl RL and Subramaniam RM: Quantitative PET/CT in clinical practice: Assessing the agreement of PET tumor indices using different clinical reading platforms. Nucl Med Commun 39: 154-160, 2018.

18. Cazzato RL, Garnon J, Shaygi B, Koch G, Tsoumakidou G, Caudrelier J, Addeo P, Bachellier P, Namer IJ and Gangi A: PET/CT-guided interventions: Indications, advantages, disadvantages and the state of the art. Minim Invasive Ther Allied Technol 27: 27-32, 2018.

19. Stecco A, Buemi F, Cassarà A, Matheoud R, Sacchetti GM, Arnulfo A, Brambilla $\mathrm{M}$ and Carriero A: Comparison of retrospective PET and MRI-DWI (PET/MRI-DWI) image fusion with PET/CT and MRI-DWI in detection of cervical and endometrial cancer lymph node metastases. Radiol Med (Torino) 121: 537-545, 2016.

20. Catalano OA, Daye D, Signore A, Iannace C, Vangel M, Luongo A, Catalano M, Filomena M, Mansi L, Soricelli A, et al: Staging performance of whole-body DWI, PET/CT and PET/MRI in invasive ductal carcinoma of the breast. Int J Oncol 51: 281-288, 2017.

21. Gaeta CM, Vercher-Conejero JL, Sher AC, Kohan A, Rubbert C and Avril N: Recurrent and metastatic breast cancer PET, PET/CT, PET/MRI: FDG and new biomarkers. Q J Nucl Med Mol Imaging 57: 352-366, 2013.

This work is licensed under a Creative Commons Attribution-NonCommercial-NoDerivatives 4.0 International (CC BY-NC-ND 4.0) License. 